\title{
The Management of Anaesthesia in Caesarean Section of Pregnant Women with COVID-19
}

\author{
Gunes Ozlem Yıldı' , [MD] \\ ORCID: 0000-0002-4557-9517 \\ Zafer Cukurova', [MD] \\ ORCID: 0000-0002-8893-3977 \\ Gulsum Oya Hergunsel', [MD] \\ ORCID: 0000-0003-3218-0029 \\ Elif Marangoz', [MD] \\ ORCID: 0000-0003-2792-4548
}

'Department of Anesthesiology and Intensive Care, University of Health Sciences Istanbul, Bakirkoy Dr. Sadi Konuk Training and Research Hospital, Istanbul, Turkey.

\section{Corresponding Author: Güneș Özlem Yıldız}

Department of Anesthesiology and Intensive Care, University of Health Sciences Istanbul, Bakirkoy Dr. Sadi Konuk Training and Research Hospital, Istanbul, Turkey Phone: +90212 4147171

E-mail: drgunesim@hotmail.com

https://doi.org/10.32552/2021.ActaMedica.485

\section{n ABSTRACT Con}

Objective: In this study, we aimed to investigate the effect of preoperative clinical status on the choice of anesthesia method and the postoperative maternal and fetal outcomes in the caesarean section of pregnant women with COVID-19 related acute respiratory distress syndrome.

Materials and Methods: Pregnant women with COVID-19 related acute respiratory distress syndrome, for whom caesarean sections were planned, were included in this retrospective study. Clinical features, preoperative findings and treatments, maternal and fetal outcomes in the preoperative period, intensive care needs, and hospital stay were evaluated.

Results: Ten patients who underwent caesarean section were included in the study. The mean age of the patients was $34 \pm 1.82$ and the mean gestational week was $33.1 \pm 3.88$. Chronic disease was present in $44.4 \%$ of them. Sixty percent of the patients were admitted to the postoperative intensive care unit. Eighty percent of the newborns were preterm and half of the newborns needed intensive care unit hospitalization.

Conclusion: The clinical features of pregnant women with COVID-19 are similar to those reported for non-pregnant adults with COVID-19, but respiratory parameters of pregnant women with COVID-19 related acute respiratory distress syndrome deteriorate rapidly and should be monitored closely. Preoperative clinic status and oxygenation are the most important determinants in the selection of anesthesia type for the caesarean section.

Keywords: Acute respiratory distress syndrome, caesarean section, COVID-19 pandemic, intensive care units, pregnancy

Received: 11 August 2020, Accepted: 11 October 2020,

Published online: 5 February 2021

\section{INTRODUCTION}

Coronavirus disease 2019 (COVID-19) has been declared a pandemic by the World Healt Organization (WHO) [1]. COVID-19 cases were characterized by complaints such as cough, dyspnea, fever, nausea and lung infection. When epidemiologically examined, it was seen that all of these patients had a history of visiting Wuhan (Hubei, China) seafood and wild animal market. When the throat swab samples of the patients evaluated by the disease control committee of the People's Republic of China were examined, the detected pathogen was called SARS Cov 2 virus, and the name of the disease was called COVID-19 by WHO.

SARS Cov 2 also has 85\% similarity with other coronaviruses, such as SARS (severe acute respiratory syndrome) and MERS (Middle East respiratory syndrome) viruses. SARS and MERS viruses markedly caused poor fetal and maternal outcomes such as preterm birth, stillbirth, respiratory complications, and maternal mortality. 
However, there is no evidence of a vertical transition from mother to baby $[2,3]$.

Maternal physiological adaptations do not only make the pregnant woman more vulnerable to cell-mediated viral infections such as COVID-19 but also more prone to rapid cardiopulmonary decompensation due to decreased cardiac and pulmonary reserves. The delivery process including physiological adaptation mechanisms a catecholaminergic fluctuation, release of inflammatory mediators in the endothelium, and significant fluid changes between the interstitial, intracellular and intravascular compartments [4]. In case of severe systemic infection, these physiological changes can exacerbate the complex inflammatory condition that leads to a higher potential for endothelial dysfunction, pulmonary edema, myocardial edema, and cardiac dysfunction. It is assumed that the risk of COVID-19 infection is increased or has a severe course in pregnant women compared to other adults, and it has been observed that disease severity in COVID-19 infection is lower compared to SARS1 and MERS, though it is more infective [3].

Therefore, it should be taken into consideration that pregnant women with COVID 19, and especially pregnant women with COVID-19-related severe acute respiratory distress syndrome (ARDS), can be rapidly decompensated and these patients should be managed with a multidisciplinary approach [4]. Determination of the severity of maternal clinical condition, preparation for birth, close monitoring of the fetus and mother, management of the preferred anesthesia and postnatal monitoring of the mother and newborn in the ward or intensive care unit, if necessary, are important for optimum maternal and fetal clinical outcomes [5].

In our study, we aimed to investigate the effect of preoperative clinical status on the choice of anesthesia method and postoperative maternal and fetal outcomes following caesarean section in COVID-19 possible pregnant women in the presence of COVID-19-related ARDS.

\section{MATERIAL and METHODS}

Our study was approved by Bakırköy Sadi Konuk Training and Research Hospital ethics committee. (2020\288) The results of pregnant women with possible COVID-19 infection, aged above 18 years, who presented to the Gynecology and Obstetrics Clinic of our hospital between April 2020 and June 2020, and who were decided to have caesarean section because of preeclampsia, fetal distress, malpresentation, rapidly developing respiratory distress and/or reduced saturation were evaluated. All clinical data were collected after obtaining written informed consent from our patients. The SARS-CoV-2 quantitative RT-PCR (polymerase chain reaction (PCR) test, rapid antibody test, and thorax tomography were used preoperatively to diagnose patients who had a history of contact and /or presented with COVID-19 symptoms. History of contact with COVID-19 patients, symptoms, demographic data, American Society of Anesthesiologists (ASA) scores, and the presence of chronic disease were questioned and recorded in the preoperative period.

Before the patients were transferred to the operating room, all employees were provided with personal protective equipment (disposable overalls, N95 masks, medical gloves, glasses, and a protective shields). Anesthetic interventions, endotracheal intubation, and regional anesthesia were performed by anesthesiologists. Patients were admitted to the operating room with a mask. Standard monitoring electrocardiogram, pulse oximetry, noninvasive blood pressure measurement was provided. In our patients, we primarily preferred regional anesthesia both to reduce aerosol transmission and to avoid the pulmonary complications of COVID-19, but general anesthesia was also performed in cases of patient's refusal, severe maternal respiratory distress, and fetal distress.

For spinal anesthesia, a single dose of hyperbaric bupivacaine that is calculated according to the patient's height, weight, and age, was administered inserting a 25 Gauge Quincke spinal needle in the L3-4 interspace in sitting position.

In the general anesthesia procedure, rapid sequence standardized intubation method was planned after $100 \%$ preoxygenation. The patients were intubated by clamping the end part of the endotracheal tube in the aerosol box and attached to the anesthesia machine. A filter was placed in the inspiratory and expiratory circuit lines of the anesthesia machine. Propofol at a dose of $2-3 \mathrm{mg} / \mathrm{kg}$ and rocuronium at a dose of $0.6 \mathrm{mg} / \mathrm{kg}$ were administered for induction of general anesthesia. Sevoflurane MAC 1-1.5 was used for anesthesia maintenance. 
Preoperative maternal blood gas, fetal birth weight, fetal 1. and 5th Minute APGAR scores, fetal blood gas, and postoperative fetal SARS-CoV-2 quantitative RT-PCR (polymerase chain reaction (PCR) test result, the need for intensive care hospitalization, the duration of intensive care stay and the hospital stay of the mother and baby were recorded.

\section{RESULTS}

In our study, 10 caesarean section cases with possible COVID-9 were included. The mean age of patients was $34 \pm 1.82$, The Body Mass Index (BMI) ( $\mathrm{kg} / \mathrm{m} 2$ ) was $31.71 \pm 8.99$. Chronic disease was present in $44.4 \%$. Three (30\%) patients were ASA3 and 7 (70\%) were ASA2.

Demographic data and clinical status of the patients and COVID-19 diagnostic test results are shown (Table 1).

Spinal anesthesia was applied to four patients (40\%) and general anesthesia was applied to six patients (60\%). Three patients undergoing general anesthesia had serious respiratory distress, dyspnea, and low saturation at admission to the operating room, and they were admitted under oxygen support. One of our 2 patients, who had a history of contact but no symptoms, had lung CT findings (Table 1, Figure 1), and the other one had rapid antibody test positivity.

The mean lymphocyte count of the patients was $1105 \pm 292$, the mean platelet count was $177250 \pm 35845$. The $\mathrm{C}$-reactive Protein (CRP) values of all patients were found to be increased on the first day (> 5mg / L) (Table 2). There were preoperative hypotension, tachycardia, low saturation, and hemodynamic instability in patients 4 and 8 .

All pregnant women had single pregnancies. All of the babies were girls. No intrapartum fetal death or neonatal death was observed. 7 of the newborns were preterm and the birth weight of 6 newborns was $<2500$ grams (Table 3 ).

Table 1. Demographic Characteristics and Clinical Symptoms of 10 Possible COVID-19 Pregnant Women

\begin{tabular}{|c|c|c|}
\hline Baseline Characteristics & & Number of Patients (\%) \\
\hline \multirow[t]{4}{*}{ Preoperative Comorbidities } & Diabetes & $1(10)$ \\
\hline & Hypothyroidism & $1(10)$ \\
\hline & Aorta aneurysm \& Pulmonary hypertension & $1(10)$ \\
\hline & Cardiomegaly & $1(10)$ \\
\hline \multirow[t]{13}{*}{ Admission Symptoms } & Cough & $7(70)$ \\
\hline & Shortness of breath & $5(50)$ \\
\hline & Fever* & $3(30)$ \\
\hline & SARS-CoV-2 quantitative RT-PCR test & $3(30)$ \\
\hline & Serum test for SARS-CoV-2 IgG and IgM & $2(20)$ \\
\hline & Lung imaging findings ${ }^{\dagger}$ & $8(80)$ \\
\hline & Bilateral and Multilobe & $8(80)$ \\
\hline & Patchy & $4(40)$ \\
\hline & Nodular & $4(40)$ \\
\hline & Ground-Glass Opacification & $8(80)$ \\
\hline & Pleural Effusion & $3(30)$ \\
\hline & Atelectasis & $2(20)$ \\
\hline & Cardiomegaly & $3(30)$ \\
\hline \multirow[t]{6}{*}{ Caesarean Indication } & Fetal Distress & $2(20)$ \\
\hline & Preeclampsia & $4(40)$ \\
\hline & Preeclampsia and severe respiratory distress & $1(10)$ \\
\hline & Severe Respiratory Distress & $2(20)$ \\
\hline & Malpresentation & $1(10)$ \\
\hline & Previous Caesarean Delivery & $1(10)$ \\
\hline
\end{tabular}

*Defined as a temperature of greater than $37.5^{\circ} \mathrm{C} ;{ }^{+}$Chest imaging findings were evaluated by the radiologist 

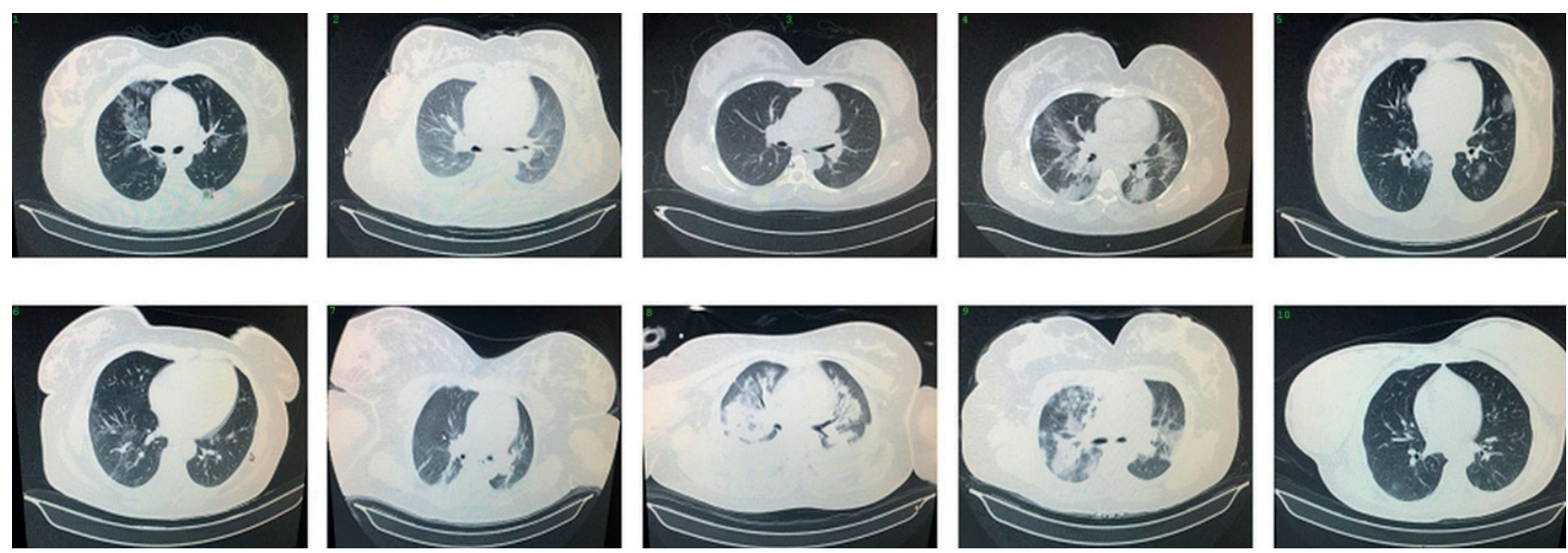

Figure 1. Lung CT Findings of Patients

'Patient 1: Bilateral ground-glass opacities with multiple round configurations

2Patient 2: Ascending aorta aneurysmatic dilated. Bilateral mild pleural effusion, pleuroparancimal fibrotic density, subsegment atelectasis

${ }^{3}$ Patient 3: Bilateral clear lung fields with no obvious ground-glass opacities.

${ }^{4}$ Patient 4: Multiple bilateral ground-glass opacities, prominent on the left

${ }^{5}$ Patient 5: Multiple bilateral ground-glass opacities

${ }^{6}$ Patient 6: Bilateral central-peripheral nodular ground-glass opacities

7Patient 7: Atelectasis areas, bilateral minimal pleural effusion

${ }^{8}$ Patient 8: Cardiomegaly, bilateral pleural effusion and central consolidation-ground-glass density

${ }^{9}$ Patient 9: Multiple bilateral ground-glass opacities

${ }^{10}$ Patient 10: Bilateral pleural effusion, atelectasis areas and pleuroparachimal fibrotic band formations

Table 2. Laboratory Results of Possible COVID-19 Pregnant Women

\begin{tabular}{|l|c|c|c|c|c|c|c|c|c|c|}
\hline & Pat*.1 & Pat.2 & Pat.3 & Pat.4 & Pat.5 & Pat.6 & Pat.7 & Pat.8 & Pat.9 & Pat.10 \\
\hline LDH $^{+}$Day1 & 329 & 233 & 214 & 313 & 245 & 254 & 330 & 325 & 350 & 299 \\
\hline LDH Day5 & 360 & 276 & 255 & 335 & 280 & 380 & 494 & 446 & 420 & 264 \\
D-Dimer Day1 & 1.57 & 0.73 & 0.55 & 0.74 & 0.5 & 1.41 & 6.09 & 1.79 & 0.04 & 2.12 \\
\hline D-Dimer Day5 & 1.3 & 0.26 & 1.42 & 1.1 & 1.3 & 2.02 & 3.1 & 1.29 & 3.35 & 2.05 \\
\hline CRP Day1 & 56 & 29 & 6 & 153.2 & 6.99 & 9.3 & 101 & 45.7 & 130 & 141 \\
\hline CRP Day5 & 36 & 13 & 14 & 115 & 9 & 89 & 298 & 56 & 186 & 93.3 \\
Procalcitonin-Day1 & 0.24 & 0.12 & 0.08 & 0.68 & 0.03 & 0.04 & 0.62 & 0.12 & 0.24 & 0.7 \\
Procalcitonin-Day5 & 0.12 & 0.11 & 0.42 & 0.49 & 0.5 & 0.03 & 1.9 & 0.3 & 4.42 & 0.29 \\
Ferritin Day1 & 137.8 & 192 & 8.9 & 94 & 34.2 & 33 & 41.3 & 8.8 & 59.6 & 39 \\
Ferritin Day5 & 70.8 & 37.3 & 6.7 & 70.9 & 28.6 & 94 & 41 & 40.8 & 40 & 38 \\
Ph & 7.38 & 7.4 & 7.31 & 7.29 & 7.34 & 7.35 & 7.33 & 7.09 & 7.27 & 7.38 \\
Po2 & 62.5 & 75 & 65 & 74.8 & 80 & 52 & 73.4 & 42 & 56 & 90 \\
Pao2/Fio2 & 125 & 150 & 108 & 123 & 160 & 86 & 122 & 60 & 93 & 180 \\
\hline Spo2 & 92 & 90 & 92 & 81 & 94 & 88 & 90 & 70 & 85 & 95 \\
Pco2 & 36.2 & 35.6 & 47 & 51.8 & 45 & 42 & 38 & 71.8 & 21 & 40 \\
\hline Lactate & 2 & 1 & 1.3 & 1 & 1 & 1 & 1.1 & 4.2 & 1 & 2 \\
\hline
\end{tabular}

*Patient; ${ }^{\dagger}$ Lactat Dehidrogenaz; ${ }^{\ddagger} \mathrm{C}-$ reactive protein concentration (mg/L)

\section{DISCUSSION}

The clinical features of the patients in our study were similar to that non-pregnant adults with COVID-19 infection as reported previously [6,7]. Among our patients' complaints at the time of presentation, dry cough ranked first and fever ranked second. Laboratory tests showed that lymphocyte count decreased, the neutrophil rate increased, C-reactive protein and D-dimer increased in accordance with the literature [7][8]. The ferritin elevation observed in normal adults was not observed in our patients. We think that this is due to the already low level 
Table 3. Postoperative Maternal and Fetal Outcomes of Possible COVID-19 Pregnant Women

\begin{tabular}{|l|c|c|c|c|c|c|c|c|c|c|}
\hline & Pat*1 & Pat.2 & Pat.3 & Pat.4 & Pat.5 & Pat.6 & Pat.7 & Pat.8 & Pat.9 & Pat.10 \\
\hline Maternal ICU bt & No & Yes & No & Yes & No & No & Yes & Yes & Yes & Yes \\
Maternal ICU Day & 0 & 1 & 0 & 15 & 0 & 0 & 2 & 3 & 10 & 3 \\
Fetal Weight & 2380 & 665 & 3635 & 2305 & 1500 & 2720 & 2660 & 780 & 1100 & - \\
APGAR 1 & 7 & 7 & 9 & 7 & 7 & 7 & 7 & 0 & 6 & 7 \\
APGAR 5 & 9 & 8 & 10 & 8 & 8 & 9 & 9 & 6 & 8 & 9 \\
Fetal ICU & No & Yes & Yes & Yes & Yes & No & No & Yes & No & No \\
Fetal ICU Day & 0 & 5 & 3 & 11 & 5 & 0 & 0 & 25 & 0 & 0 \\
Maternal Length of HS $^{\ddagger}$ & 5 & 20 & 8 & 21 & 13 & 11 & 10 & 15 & 17 & 12 \\
\hline
\end{tabular}

${ }^{*}$ Patient; ${ }^{\dagger}$ Intensive Care Unit; ${ }^{\ddagger}$ Hospital Stay

of ferritin associated with physiological anemia of pregnancy.

Caesarean section indications of the patients in our study: three of our patients had preeclampsia and respiratorydistress. Sincetheyhad severe respiratory distress and hypoxemia, they were transferred to the operation room under oxygen support. When they were transferred to the operation room under oxygen support, SpO2 was $<\% 85$. Severe dyspnea, respiratory distress, tachypnea, or hypoxia are warning signs for the severity of the disease [9]. In the severe form of the disease, there is an increased risk of morbidity and mortality for the mother and baby. Other indications were fetal distress, previous history of caesarean section, and malpresentation. The choice of anesthesia method in caesarean cases is critical for pregnant women with COVID-19 since the lung is the main target organ of the SARS $\mathrm{CoV}-2$ virus. There is no evidence that regional anesthesia is contraindicated in the presence of coronavirus. Although spinal anesthesia is our first choice to reduce pulmonary complications due to intubation, to prevent transmission of the infection to medical personnel and the increase of viral load in the environment, general anesthesia was applied to 6 patients due to reasons such as patient's refusal, severe respiratory distress, and hypoxemia. Two of the 4 patients who underwent spinal anesthesia, were hospitalized in the ICU due to postoperative respiratory distress and hypoxemia.

Thorax tomography of pregnant women with possible COVID-19 revealed diffuse and bilateral opacities, irregular lung consolidation areas, diffuse ground-glass appearance, and pleural effusion in some patients as observed in other publications [10-12].

COVID-19 pneumonia forms an ARDS clinic with normal respiratory compliance, hypoxemia, and its incidence has been reported to be $15.6 \%-31 \%$ $[13,14]$. The onset time of COVID-19-related ARDS has been reported to be 8-12 days in different studies $[1,13,14]$. Based on their experience, experts from the Chinese national healthcare commission developed a standard treatment protocol for COVID-19 and divided it into three categories according to the COVID-19-related ARDS oxygenation index (mild $200 \mathrm{mmHg} \leq \mathrm{PaO}_{2}$ $/ \mathrm{FiO}_{2}<300 \mathrm{mmHg}$, mild-moderate $150 \mathrm{mmHg} \leq$ $\mathrm{PaO}_{2} / \mathrm{FiO}_{2}<200 \mathrm{mmHg}$, and moderate-severe $\mathrm{PaO}_{2} / \mathrm{FiO}_{2}<150 \mathrm{mmHg}$ ) [15]. According to this classification, 7 (70\%) of our patients were $\mathrm{PaO}_{2} /$ FiO $<150 \mathrm{mmHg}$ and moderate-severe ARDS, other $3(30 \%)$ patients were in $\mathrm{PaO}_{2} / \mathrm{FiO}_{2}<200 \mathrm{mmHg}$ and were in the medium-light ARDS category. The main factors for us to prefer general anesthesia over spinal anesthesia in our patients were the presence of severe respiratory distress and $\mathrm{PaO}_{2} /$ $\mathrm{FiO}_{2}$ value of $<150$. Eight of our patients ( $80 \%$ ) were in stage $2 \mathrm{~b}$ (pulmonary involvement with hypoxia) according to the definition of Siddiqi et al. (Stage 2: shortness of breath, hypoxia $\mathrm{PaO}_{2} / \mathrm{FiO}_{2}<300$, normal procalcitonin values and abnormal chest imaging findings). At this stage, patients develop a viral pneumonia with cough, fever, and possibly hypoxia [16].

In a study conducted by Chen et al. involving 9 cases, it was reported that none of the patients needed hospitalization in the intensive care unit and needed mechanical ventilation [12,17]. However, 6 of the patients in our study had COVID-19-related ARDS, and were admitted in ICU and mechanical ventilation was required in these patients following the caesarean section. 4 of 6 patients with ICU hospitalization had pre-existing comorbidity.

In a study conducted by Liu et al., 6 patients were admitted to intensive care unit with ARDS requiring 
intubation and mechanical ventilation and had multiple organ dysfunction syndrome (MODS), including acute hepatic insufficiency, acute renal failure, and septic shock [18]. Our patients had a clinic of COVID-19-related ARDS and needed mechanical ventilation, but multiple organ failure or mortality was not observed.

Perinatal 2019-nCoV infection can have adverse effects in newborns, which can cause problems such as fetal distress, preterm labor, respiratory distress, thrombocytopenia accompanied by abnormal liver function, and even death [11]. Eight of the newborns were preterm ( $<37$ weeks) and 6 newborns had a birth weight of $2500 \mathrm{gram}$. Five (50\%) of the newborns were hospitalized in the neonatal intensive care unit. The APGAR scores were between 7 and 9. Our study supports the literature in that it involved the presence of fetal distress, SGA newborns, and ICU hospitalizations of newborns.

In a meta-analysis, preterm delivery was reported to be the most common negative pregnancy outcome in $>90 \%$ of pregnant women hospitalized due to COVID-19 pneumonia, and an increase in rates of preterm delivery, preeclampsia, and caesarean section, was reported. There is no clinical evidence of vertical transmission from mother to baby [19]. In our study, 4 of our patients had preeclampsia and 8 had a premature delivery. SARS-CoV-2 RT-PCR test was performed in the newborns and SARS-CoV-2 RT-PCR test results of all babies were found to be negative. We think that this will shed light on the literature in terms of vertical transmission.
SARS-CoV-2 RT-PCR tests were found to be negative twice in all of our patients, their clinical symptoms diminished and they were discharged after $16 \pm 5.35$ days similar to the 16-day hospital stay in the study conducted by LIU et al. Mortality was not observed in any of our patients.

Our study has several limitations. There was a low number of patients in our study. Prospective studies with larger samples are needed to gain a more comprehensive understanding of COVID-19related ARDS during pregnancy. Secondly, the early results of the newborns are given. The long-term results of the patients should be investigated.

In conclusion, the clinical features of pregnant women with COVID-19 are similar to those reported for non-pregnant adults with COVID-19. Respiratory parameters of pregnant women with COVID-19related ARDS may deteriorate rapidly and should be monitored closely. Preoperative clinic status and oxygenation are the most important determinants in the selection of anesthesia management for the caesarean section.

\section{ACKNOWLEDGMENTS}

The authors have declared that no funding was received for this work.

\section{CONFLICTS OF INTEREST}

The authors have declared that no conflict of interest exists. 
[1] Ashokka B, Loh $\mathrm{MH}$, Tan $\mathrm{CH}$, et al. Care of the pregnant woman with coronavirus disease 2019 in labor and delivery: anesthesia, emergency cesarean delivery, differential diagnosis in the acutely ill parturient, care of the newborn, and protection of the healthcare personnel. Am J Obstet Gynecol. 2020; 223(1): 66-74.e3.

[2] Wang L, Wang Y, Ye D, Liu Q. Review of the 2019 novel coronavirus (SARS-CoV-2) based on current evidence. Int J Antimicrob Agents. 2020; 55(6): 105948.

[3] Sun P, Lu X, Xu C, Sun W, Pan B. Understanding of COVID-19 based on current evidence. J. Med. Virol. 2020; 92(6): 548551.

[4] SchnettlerWT, AI AhwelY, Suhag A. Severe acute respiratory distress syndrome in coronavirus disease 2019-infected pregnancy: obstetric and intensive care considerations. Am J Obstet Gynecol MFM. 2020; 2(3): 100120.

[5] Panahi L, Amiri M, Pouy S. Risks of Novel Coronavirus Disease (COVID-19) in Pregnancy; a Narrative Review. Arch Acad Emerg Med. 2020; 8(1): e34.

[6] Huang C, Wang Y, Li X, et al. Clinical features of patients infected with 2019 novel coronavirus in Wuhan, China. Lancet. 2020; 395(10223): 497-506.

[7] Wong SF, Chow KM, Leung TN, et al. Pregnancy and perinatal outcomes of women with severe acute respiratory syndrome. Am J Obstet Gynecol. 2004; 191(1): 292-297.

[8] Yu N, Li W, Kang Q, et al. Clinical features and obstetric and neonatal outcomes of pregnant patients with COVID-19 in Wuhan, China: a retrospective, single-centre, descriptive study. Lancet Infect Dis. 2020; 20(5): 559-564.

[9] Breslin N, Baptiste C, Gyamfi-Bannerman C, et al. Coronavirus disease 2019 infection among asymptomatic and symptomatic pregnant women: two weeks of confirmed presentations to an affiliated pair of New York City hospitals. Am J Obstet Gynecol MFM. 2020; 2(2): 100118.
[10] Chung M, Bernheim A, Mei X, et al. CT imaging features of 2019 novel coronavirus (2019-NCoV). Radiology. 2020; 295(1): 202-207.

[11] Zhu H, Wang L, Fang $C$, et al. Clinical analysis of 10 neonates born to mothers with 2019-nCoV pneumonia. Transl Pediatr. 2020; 9(1): 51-60.

[12] Liu D, Li L, Wu X, et al. Pregnancy and perinatal outcomes of women with coronavirus disease (COVID-19) Pneumonia: A preliminary analysis. Am J Roentgenol. 2020; 215(1): 127-132.

[13] Wang D, Hu B, Hu C, et al. Clinical Characteristics of 138 Hospitalized Patients with 2019 Novel CoronavirusInfected Pneumonia in Wuhan, China. JAMA - J Am Med Assoc. 2020; 323(11): 1061-1069.

[14] Zhou F, Yu T, Du R, et al. Clinical course and risk factors for mortality of adult inpatients with COVID-19 in Wuhan, China: a retrospective cohort study. Lancet. 2020; 395(10229): 1054-1062.

[15] Li X, Ma X. Acute respiratory failure in COVID-19: Is it "typical" ARDS? Crit. Care. 2020; 24(1): 198.

[16] Siddiqi HK, Mehra MR. COVID-19 illness in native and immunosuppressed states: A clinical-therapeutic staging proposal. J. Hear. Lung Transplant. 2020; 39(5): 405-407.

[17] Chen H, Guo J, Wang C, et al. Clinical characteristics and intrauterine vertical transmission potential of COVID-19 infection in nine pregnant women: a retrospective review of medical records. Lancet. 2020; 395(10226): 809-815.

[18] Liu Y, Chen H, Tang K, Guo Y. Clinical manifestations and outcome of SARS-CoV-2 infection during pregnancy. J. Infect. 2020.

[19] Di Mascio D, Khalil A, Saccone G, et al. Outcome of coronavirus spectrum infections (SARS, MERS, COVID-19) during pregnancy: a systematic review and meta-analysis. Am J Obstet Gynecol MFM 2020; 2(2): 100107. 